\title{
ASSESSING AND PREDICTING THE IMPACT OF COWLSHAKE IN CONVERTIBLE CARS ON SUBJECTIVE COMFORT
}

\author{
Harald Kolrep \\ Kolrep-Rometsch, Human Factors Consultants \\ Berlin, Germany \\ E-mail: hko@kolrep-rometsch.de
}

\begin{abstract}
Summary: Torsional oscillations of the car body of convertible cars give rise to cowlshake of the wind-shield frame and the rear-view mirror, the dashboard, steering wheel, and seats. Drivers can perceive these oscillation which might impair subjective comfort and driving safety. Intensity reaches from just noticeable movements up to annoying shake of the steering wheel and distorted view in the mirror. A method to assess drivers' subjective comfort is suggested which allows to determine a functional relation between objective parameters of cowlshake and subjective comfort. This study is a first step towards simulation of cowlshake which includes prediction of comfort impairment.
\end{abstract}

\section{INTRODUCTION}

A series of empirical studies was to investigate possible relations between objective parameters of cowlshake in roadsters and subjective comfort. The perception of cowlshake in ride sessions is influenced by traffic situation and noise, influencing factors that cannot be controlled and reproduced in experimental situations. Laboratory situation would allow exact control of influencing factors, but realistic simulation of noise and traffic is complex and expensive. We developed an mixed experimental design of ride sessions and laboratory investigations on a test rig. The study has two parts:

Subjective assessment of cowlshake. A semantic differential seems appropriate to assess subjective impairment of comfort related to cowlshake. Three series of ride session were used to develop and validate a questionnaire which used adjective contrasts for subjective assessment of cowlshake and which should differentiate cars and road conditions.

Tactile and visual perception of cowlshake. For a more detailed analysis of perceptual thresholds and human sensitivity for cowlshake, tests were conducted on a 4 poster hydropulse test rig. We used the psychophysical method of direct scaling (Stevens, 1975) to investigate human tactile perception of steering wheel shake and visual perception in rear view mirror shake.

\section{SUBJECTIVE ASSESSMENT OF COWLSHAKE IN RIDE SESSIONS}

The first step for parameter identification was a simultaneous measurement of subjective and objective data during ride sessions. 30 subjects drove 3 different cars on 3 different road conditions. Cars were of the same type, but modified to obtain low, intermediate, and high cowlshake. 


\section{Developing a semantic differential}

The semantic differential method has been developed by Osgood (1952, Osgood, May \& Miron, 1975) for the analysis of meaning. It has frequently been used in other domains of psychology. The method is particularly useful to assess subjective judgements and attitudes (Schäfer, 1983). In the driving context it has successfully been used to assess noise (Kuwano et al, 1994, Takao \& Hashimoto, 1994). According to Helander \& Zhang (1997, Zhang, Helander \& Drury, 1996) the concepts of comfort and discomfort are independent entities associated with different factors. Comfort is related to well-being and aesthetics whereas discomfort has to do with biomechanics and fatigue. Therefore a multidimensional method like the semantic differential seems appropriate to asssess comfort impairment.

One of the goals was to investigate typical customers' comfort appraisal. Training of the subjects was not allowed. To obtain a usable and valid set of pairs of adjectives, three ride sessions were needed. In the first ride session open interviews led to verbal descriptions of the cowlshake phenomenon. Descriptive adjectives were extracted from the verbal reports. In the second ride session a preliminary version of the questionnaire used 23 pairs of adjectives with opposite meaning. Results were analysed regarding test criteria. Only pairs of adjectives satisfying distribution and reliability criteria were used in the final version of the questionnaire with 12 pairs of adjectives. In the third ride session the set of pairs of adjectives was validated and factor-analysed.

\section{Results}

Figure 1 shows the profile of the semantic differential ${ }^{1}$ in three different cars. Subjects can clearly differentiate between cars. Differences are statistically significant for all adjective pairs except the sixth and seventh pair. We found some significant interaction of the factors car and road conditions. The intermediate car was judged to react more to differences in road conditions than the two other cars.

The subjective appraisal had to be correlated to objective measures. We had eight channels of acceleration measurement at different positions and in different directions. For the correlation with the subjective steering wheel appraisal we used the steering wheel acceleration measurement in lateral direction. The data went through a fourier-transformation and bandfiltering into small time windows. We then counted the number of transgressions over a fixed acceleration level. The individual pairs of adjectives are factorized similar to Osgoods (1975) EPA-structure (evaluation, potency, activity). Especially for adjectives from the activity factor (the first five in figure 1) we found a correlation with 85\% explained variance with accelerations between 19 and $22 \mathrm{~Hz}$. This is a first hint that the rear wheel excitation is most important for perceived cowlshake.

\footnotetext{
${ }^{1}$ Adjectives are in the original German version. The translation in English, which is not validated, would be: goodnatured - unruly, steady - unsteady, stable - unstable, controlled - uncontrolled, pleasant - unpleasant, sporty comfortable, tight - slack, solid - hollow, sharp - blurred, direct - indirect, spartan - luxurious, cheap - stylish.
} 


\section{Steering wheel}

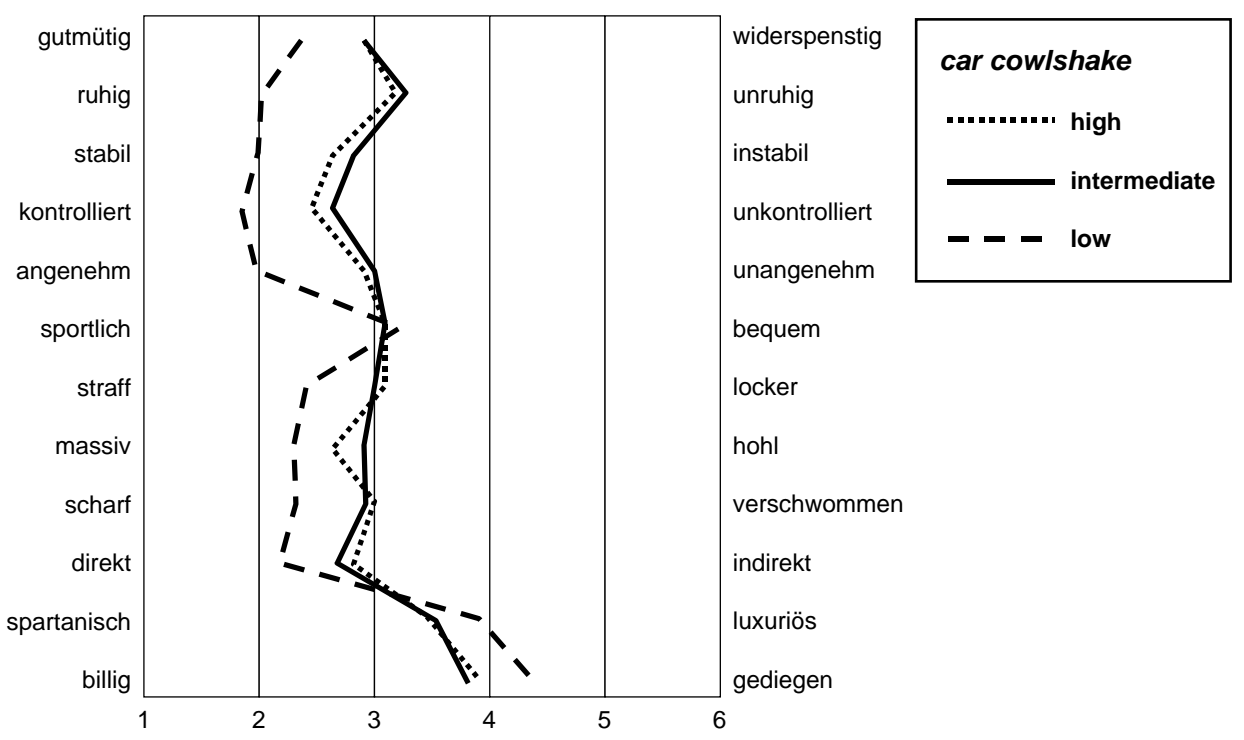

Figure 1: Profile of subjective cowlshake appraisal in different cars.

\section{HYDROPULSE INVESTIGATION OF SENSITIVITY FOR COWLSHAKE}

For a more detailed analysis of human cowlshake sensitivity, tests on a 4 poster test rig were conducted. 16 subjects had to judge the magnitude of tactile sensation of cowlshake on the steering wheel and the magnitude of visual sensation of shake in the rear view mirror. Excitation were sinus-sweeps on the rear wheel with frequency $15-22 \mathrm{~Hz}$ and $15-19 \mathrm{~Hz}$ respectively. Amplitude of excitation was clearly above threshold for all subjects. We used the method of direct magnitude estimation (Stevens 1975). Subjects had to scale their perceived magnitude of shake by calling out numbers.

Figure 2 shows on the left side the estimation of tactile cowlshake as a function of acceleration in y-direction on the steering wheel. The Stevens power-function has an exponent of 1.0868 with an explained variance of $97,4 \%$. The right side shows magnitude estimation of visual mirror shake as a function of acceleration in y-direction, measured directly on the mirror. We found an exponent of 2.7090 for the power function with an explained variance of 98,3\%. The estimated magnitude of tactile shake is nearly a linear function of acceleration whereas the visual system seems to be much more sensitive for differences in acceleration. This is probably one of the reasons why perceived mirror shake has a strong impact on subjective comfort. 

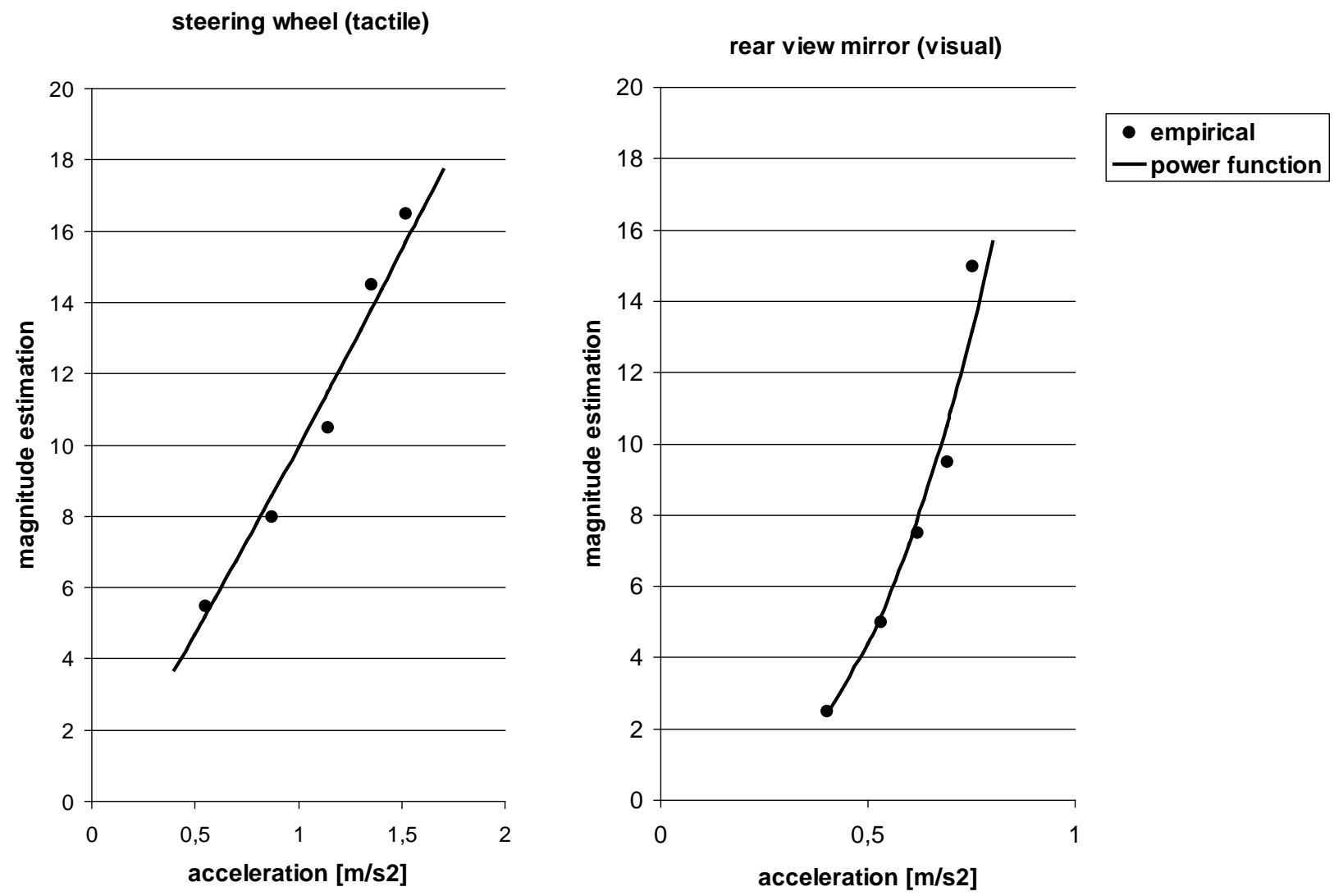

Figure 2: Magnitude estimation of cowlshake as a function of acceleration. Estimation on the steering wheel (left side) and in the rear view mirror (right side).

\section{CONCLUSIONS}

A methodology for the appraisal of the vibrational ride comfort of convertible cars has been presented in this paper. The applied measuring procedure and the hydropulse investigations have been proven as valid simplifications for the complex situation of the car under cowlshake loads on the road. The high correlation of subjective and objective cowlshake measures allow an integration of comfort impairment into simulation-models. A hybrid cowlshake model is to be developed to predict objective cowlshake and the subjective comfort impairment (Fankhauser, Riepl \& Wolauschegg, 2001). In an ongoing advanced engineering project the comfort phenomenon stuttering is being analysed. From the cowlshake investigations the next step is to introduce a total vibrational comfort tool. This tool will allow simulation and prediction of comfort in a new car concept in advance.

\section{ACKNOWLEDGEMENTS}

This research has been conducted in cooperation of Magna-Steyr Engineering (MSE) and Kolrep-Rometsch HF-Consultants. Vibrational modeling is a project of MSE. Special thanks to Christoph Fankhauser from MSE/EEN. 


\section{REFERENCES}

Fankhauser, C.; Riepl, A. \& Wolauschegg, S. (2001) Simulation of vibrational driving comfort criteria. MAGNA Steyr Engineering Symposium "Computersimulation in der Fahrzeugtechnik. (FH-Zentrum, Graz-West, 25. April 2001). Graz.

Helander, M.G. \& Zhang, L. (1997) Field studies of comfort and discomfort in sitting. Ergonomics, 40(9), 895-915.

Kuwano, S.; Namba, S; Mato, T.; Matui, M.; Miura, K. \& Imai, H. (1994) Psychologische Bewertung von Lärm in Personenwagen: Analyse nach Nationalität, Alter und Geschlecht. Zeitschrift für Lärmbekämpfung, 41, 78-83.

Osgood, C.E. (1952) The nature and measurement of meaning. Psychological Bulletin, 49, 197237.

Osgood, C.E.; May, W.H. \& Miron, M.S. (1975) Cross-Cultural Universals of affective Meaning. Urbana: University of Illinois Press.

Schäfer, B. (1983) Semantische-Differential-Technik. in: H. Feger \& J. Bredenkamp (Eds.) Datenerhebung (pp. 154-211). Göttingen: Hogrefe.

Stevens, S.S. (1975) Psychophysics. New York: Wiley.

Takao, H. \& Hashimoto, T. (1994) Die subjektive Bewertung der Innengeräusche im Fahrenden Auto - Auswahl der Adjektivpaare zur Klangbewertung mit dem Semantischen Differential. Zeitschrift für Lärmbekämpfung, 41, 72-77.

Zhang, L.; Helander, M.G. \& Drury, C.G. (1996) Identifying Factors of Comfort and Discomfort in Sitting. Human Factors, 38(3), 377-389. 